Musées, Patrimoine et Culture scientifiques et techniques

$163 \mid 2016$

janvier-février 2016

\title{
Terre-Neuve/Terre-Neuvas : retour d'expérience sur une coproduction d'expositions
}

\section{Céline Chanas}

\section{OpenEdition \\ Journals}

Édition électronique

URL : http://journals.openedition.org/ocim/1625

DOI : $10.4000 /$ ocim. 1625

ISSN : 2108-646X

Éditeur

OCIM

Édition imprimée

Date de publication : 1 janvier 2016

Pagination : 29-35

ISSN : 0994-1908

Référence électronique

Céline Chanas, «Terre-Neuve/Terre-Neuvas : retour d'expérience sur une coproduction d'expositions », La Lettre de I'OCIM [En ligne], 163 | 2016, mis en ligne le 01 janvier 2017, consulté le 19 avril 2019. URL: http://journals.openedition.org/ocim/1625; DOI : 10.4000/ocim.1625

Ce document a été généré automatiquement le 19 avril 2019

Tous droits réservés 


\section{Terre-Neuve/Terre-Neuvas : retour d'expérience sur une coproduction d'expositions}

\section{Céline Chanas}

L'entrée de l'exposition Terre-Neuve/Terre-Neuvas au musée de Bretagne : une signalétique forte.

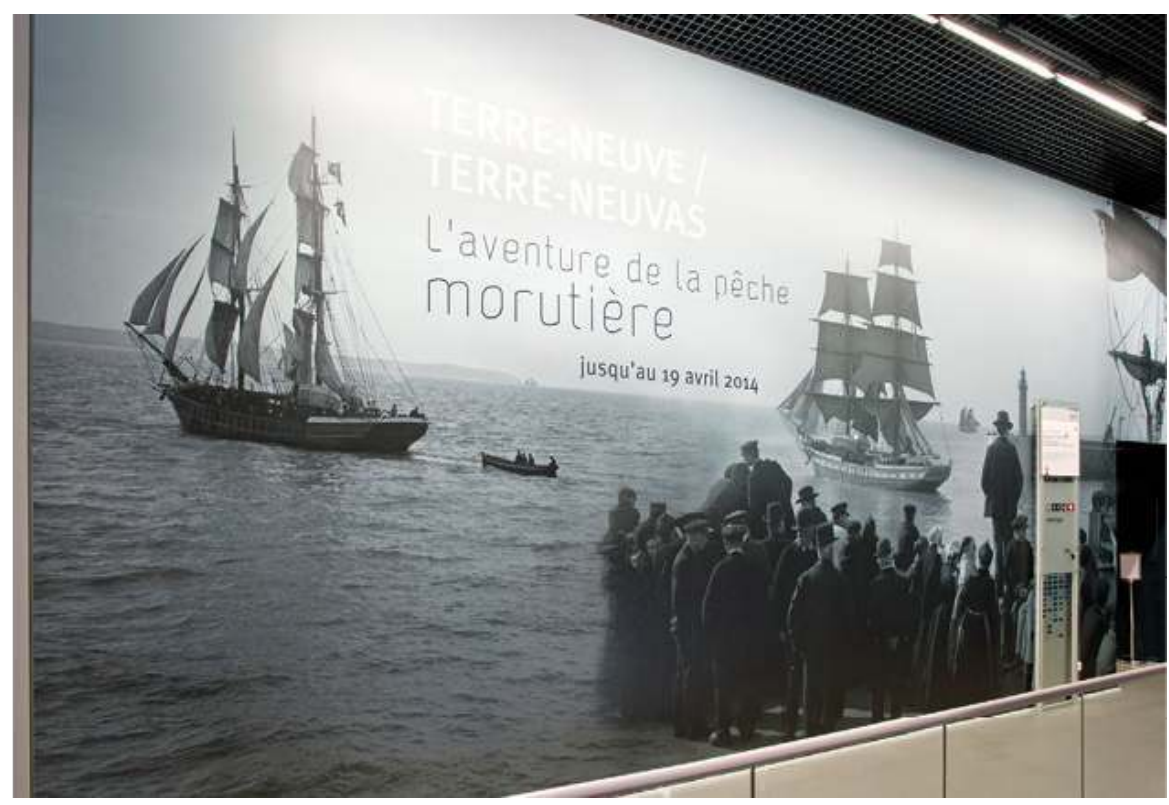

(C) Musée de Bretagne/A. Amet 


\section{Un projet, deux expositions, quatre musées sur un même thème : la Grande Pêche}

1 En 2013-2014, les musées de Bretagne à Rennes, d'Histoire à Saint-Malo, d'Art et d'Histoire à Saint-Brieuc et Granville ont présenté à leurs publics un projet ambitieux d'expositions temporaires autour de la thématique de la Grande Pêche. Ces projets ont reçu le label d'intérêt national au cours du projet, début $2014^{1}$.

2 À l'image de son sujet, Terre-Neuve/Terre-Neuvas peut être considérée pour tous les partenaires et collectivités engagées comme une exposition d'envergure, déclinée en deux volets et présentée successivement dans quatre villes entre Bretagne et Normandie.

3 Coordonné par le musée de Bretagne, ce projet de double exposition itinérante a pu voir le jour grâce à la collaboration exemplaire de quatre musées d'art, d'histoire et de société ${ }^{2}$ . Le riche fonds d'objets et de documents présentés dans les expositions est issu des collections des musées de Rennes, Saint-Brieuc, Saint-Malo et Granville, liées au thème de la Grande Pêche, enrichies de très nombreux prêts de collections publiques et de particuliers.

4 Le choix de ces villes ne doit rien au hasard. Historiquement, les ports de Saint-Brieuc, Saint-Malo et Granville ont constitué le plus grand bassin d'armement pour la pêche morutière en France. Ainsi, leurs musées possèdent des fonds d'une grande diversité sur le sujet, parfois inédits, et jamais montrés ensemble ${ }^{3}$. Quant à leurs habitants, ils entretiennent un rapport privilégié avec cette histoire collective qui irrigue souvent leur propre vécu familial. Nombre de Bretons et de Normands comptent un Terre-Neuvas dans leur arbre généalogique. Si le terme est inconnu à toute une population de la moitié est de la France, le terme est sur les côtes de l'Atlantique dans le langage courant.

5 À l'heure du bilan, il nous paraît important de dresser quelques pistes de réflexions et de partager avec la communauté des professionnels de musées l'analyse que nous portons sur cette expérience. Quels sont les enjeux de la coproduction d'expositions pour les musées aujourd'hui, et notamment ceux dont le statut est une régie directe dans le cadre juridique de collectivités territoriales? Quels avantages en termes de mutualisation de coûts, de coopération scientifique, d'ingénierie peut-on y trouver ? À quels enjeux d'un point de vue de la problématique des publics et de l'accessibilité, de tels projets peuventils répondre? A contrario, il serait illusoire de laisser penser que ces coproductions ne sont pas exemptes de difficultés : le montage juridique et financier de l'opération, la question $\mathrm{du}$ commissariat et du pilotage du projet tout comme les choix scientifiques et culturels sont autant de sujets complexes, qui pourraient parfois faire renoncer à des envies de coopération.

6 Pour autant, à la lumière du projet Terre-Neuve/Terre-Neuvas mené entre deux régions, trois départements et quatre musées, on retiendra une expérience unique, des moments forts de partage d'expériences et de sensibilités, de savoirs et de compétences diversifiées. 
La scénographie de l'exposition, réalisée par Agnès Badiche/in site, se voulait à la fois immersive et esthétique, plongeant le visiteur dans une atmosphère bleutée, évoquant le monde maritime et le travail des hommes, au travers des dispositifs audiovisuels grand format.

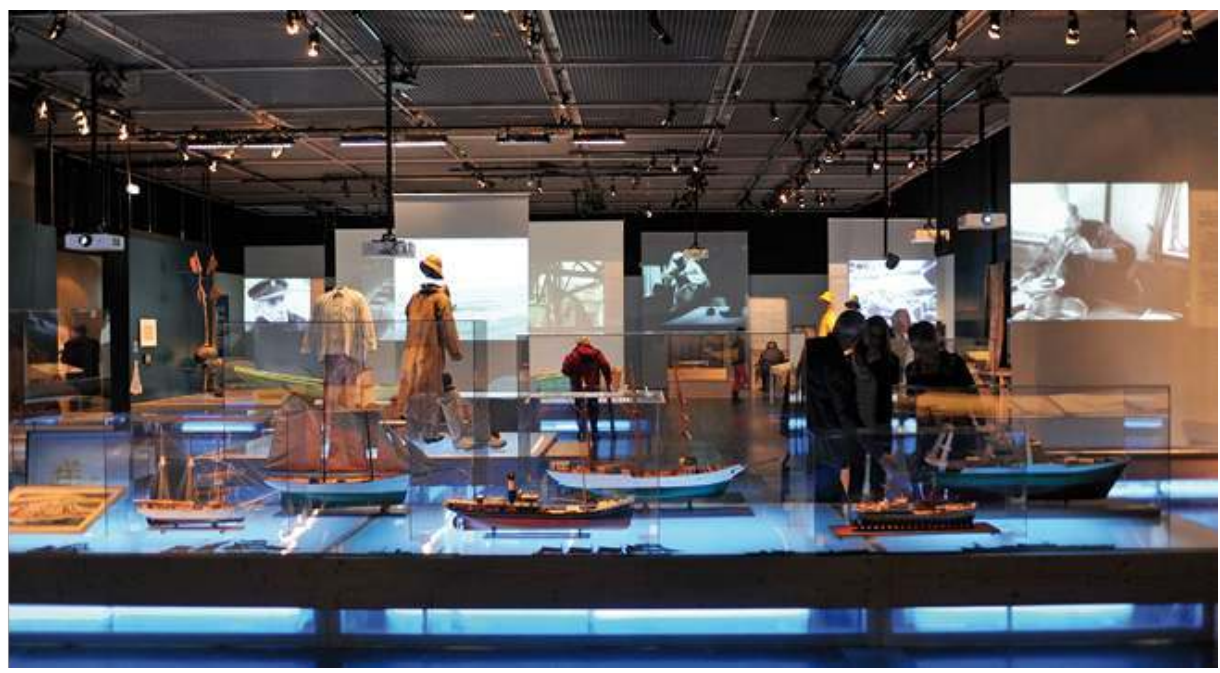

(C) Musée de Bretagne/A. Amet

\section{Les partenaires du projet : rôle et attribution}

Le projet a été initié en 2009-2010 par Pascal Aumasson, alors directeur du musée de Bretagne et proposé aux collectivités publiques tutelles après avoir emporté l'adhésion des responsables des musées partenaires pressentis, Saint-Malo, Saint-Brieuc et Granville.

Après cette première phase de concertation est signée en 2011 une convention-cadre de partenariat culturel qui "a pour objet de définir les modalités administratives et financières déterminant les engagements respectifs des partenaires dans le cadre de la préparation et la présentation des deux expositions sur le thème L'aventure Humaine des pêches à Terre-Neuve". Rennes Métropole et le musée de Bretagne assurent la maîtrise d'ouvrage du projet. Conformément à l'article 8 du Code des marchés publics, un groupement de commandes constitué des membres signataires de la convention est mis en place, chargé d'organiser, dans le respect des règles de la commande publique, la procédure des passations de marchés aboutissant au choix des prestataires. Les partenaires confient à Rennes Métropole la responsabilité des consultations nécessaires. Le musée de Bretagne assure aussi le recrutement des contractuels nécessaires au bon déroulement du projet: commissariat d'exposition, suivi technique, régie, gestion des droits... ainsi que les conventions de prêts d'objets et de partenariats divers pour l'ensemble de l'opération.

La convention-cadre comprend aussi un volet financier, avec un plan de financement global et triennal, qui précise les participations des partenaires; elle reste en revanche inaboutie sur la question de la gestion du risque, en cas de recettes moindres notamment, les collectivités n'ayant pu se mettre d'accord sur un abondement de financement le cas échéant.

La coordination scientifique et culturelle de la mise en œuvre du projet est, quant à elle, assurée conjointement, dans le cadre des instances collégiales formées par les partenaires : groupe de projet, conseil scientifique. Un règlement intérieur est mis en place qui définit ce travail collaboratif, et notamment les différentes phases de recherche, 
conception et production, ainsi que le circuit décisionnel. Le conseil scientifique réunit les directeurs et responsables scientifiques des musées partenaires, des universitaires, des spécialistes du sujet. Il a pour vocation de dresser l'état de la recherche sur le sujet envisagé, valider les orientations du scénario et proposer des pistes de collections et documents à emprunter. Il est aussi largement mis à contribution pour proposer des articles dans le cadre du projet éditorial, ouvrage de 160 pages environ sorti au moment du lancement des expositions. Il sera réuni 4 à 5 fois au cours du projet.

11 Le groupe projet est, lui, d'une autre nature: il rassemble commissariat général et scientifique ainsi que les collaborateurs directs investis sur le projet. Il se réunit environ une fois par mois, notamment dans la phase active de conception et production du projet, entre l'automne 2012 et juin 2014. Les référents des équipes des publics et de médiation culturelle y sont dès la conception associés, ce qui a permis la prise en compte dans la scénographie d'outils de médiation, d'un parcours adapté, de favoriser une large accessibilité notamment par la mise en œuvre de dispositifs audiovisuels et sonores.

La mobilité en cours de projet du chef de projet, le directeur du musée de Bretagne a aussi impliqué de repenser l'organisation du projet, avec la montée en puissance dans la coordination, d'une part du commissaire exécutif recruté, Michael Liborio, devenu véritablement chef de projet et commissaire scientifique, et une implication plus forte des directeurs d'établissements partenaires.

Satisfaisante sur le plan opérationnel, cette organisation n'a aussi sans doute pas suffisamment intégré l'échelon stratégique; des points d'étape associant les élus des collectivités partenaires sur le déroulement et les enjeux d'un tel projet auraient permis d'interroger sur le temps long d'une telle exposition le positionnement et la répartition des rôles de chacun. Là encore, la vacance de direction au musée de Bretagne durant près de 8 mois n'a pas facilité la fluidité des circuits d'information et de validation politique, ainsi que la remontée des difficultés rencontrées.

Le projet d'une itinérance outre-Atlantique est aussi envisagé, avec des repérages réalisés autour des collections à Terre-Neuve et de partenaires potentiels au Québec. Cette démarche n'a toutefois pas abouti, en raison des obstacles particulièrement importants de financement de transport et de collections. La piste de la valorisation du concept d'exposition et d'outils de médiation a alors été privilégiée. Elle s'est trouvée deux opportunités : une adaptation du projet d'exposition au musée national de la Marine à Paris en 2015-2016 avec la valorisation de la recherche et de nombreux outils de médiation et audiovisuels; une reprise de programmes multimédias pour un musée thématique à Terre-Neuve.

\section{Les enjeux scientifiques et culturels du projet}

15 Les objectifs du projet ont été bien formalisés dès le départ : ouvrir le regard des visiteurs sur un sujet d'une grande richesse; faire connaitre les données nouvelles issues des études historiques, sociologiques et ethnologiques récentes ; donner de la visibilité aux quatre musées en invitant les visiteurs à circuler d'une exposition à une autre.

Le scénario d'une double exposition s'est aussi rapidement imposé. Commun aux deux expositions, un corpus de thématiques présente en introduction les données générales du sujet : la découverte de Terre-Neuve et du Saint-Laurent, les installations outre-mer, les établissements de pêche sédentaire, le développement de la pêche errante... 
Le choix s'est porté sur la création de deux expositions-miroirs : les hommes en mer et à Terre-Neuve d'un côté; les femmes et les enfants à terre de l'autre. La première exposition, L'aventure de la pêche morutière place l'histoire sociale au cœur du sujet. Elle détaille l'économie déployée grâce à la pêche du "poisson fabuleux", l'évolution des pratiques de pêche et leurs conséquences sur les populations, les sociétés et les territoires. La seconde exposition, Le temps de l'absence, comme un contrepoint, s'intéresse à ceux restés à terre, la vie des femmes et des enfants; elle aborde les questions liées à l'absence des hommes, partis en mer, et au rôle des femmes, restées à terre. Elle met en scène la représentation littéraire et iconographique de la grande pêche, les mythes et le travail de mémoire.

Le premier volet de l'exposition a été présenté au musée de Bretagne, à Rennes sur une surface $600 \mathrm{~m}^{2}$, puis au musée d'Histoire de Saint-Malo sur $450 \mathrm{~m}^{2}$, le second volet au musée d'Art et d'Histoire de Saint-Brieuc, puis au musée d'Art et d'Histoire de Granville dans des salles d'exposition de surface quasi-identique, $380 \mathrm{~m}^{2}$ pour l'un et $425 \mathrm{~m}^{2}$ pour l'autre.

3 visiteurs sur 4 sont venus accompagnés à l'exposition, aussi bien en couple qu'en famille ou entre amis. La moitié des visiteurs indiquent avoir un lien émotionnel ou familial avec le sujet de l'exposition, et plus particulièrement parmi les visiteurs âgés de 60 ans et plus. Le temps de visite moyen est de $1 \mathrm{~h} 20$.

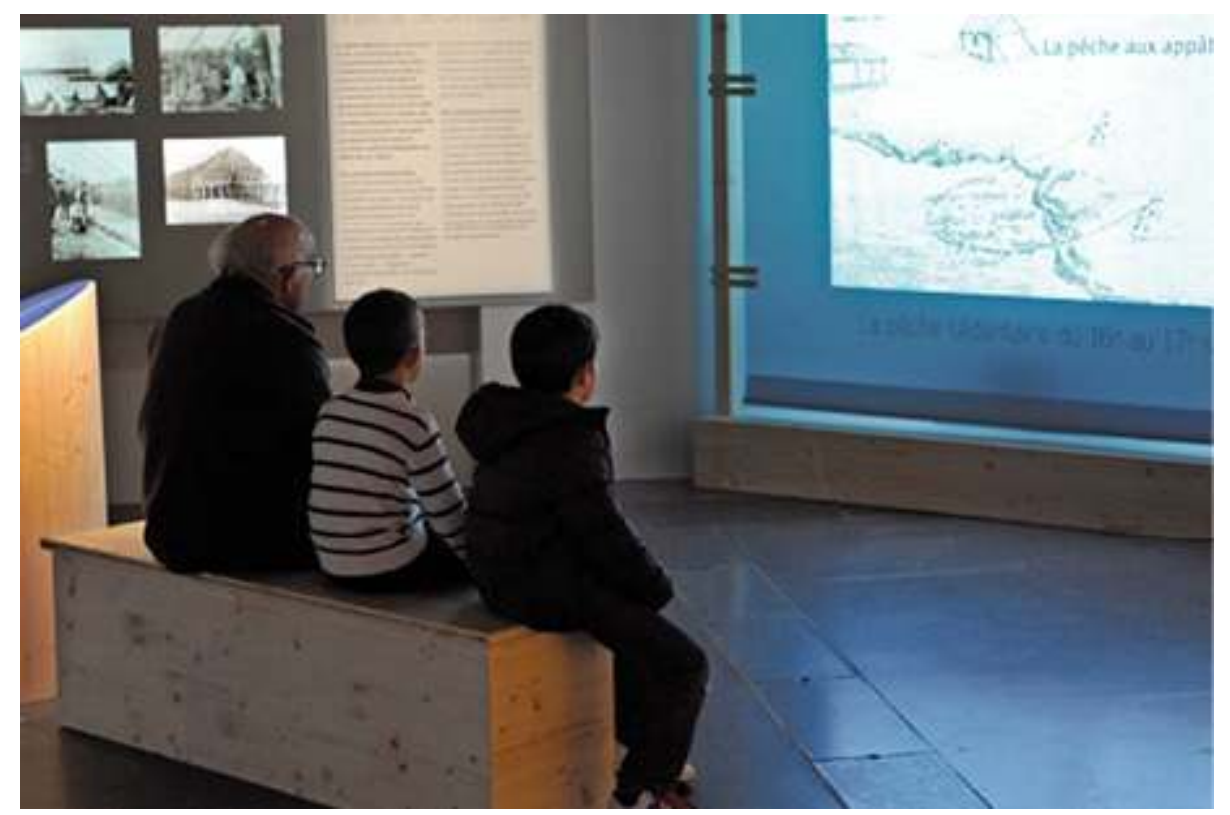

(C) Musée de Bretagne/A. Amet

19 Des collections, certaines inédites, étudiées pour ce projet ont fait l'objet de restaurations (notamment des maquettes de bateaux). Le collectage et les entretiens de mémoire orale menés posent la question de l'archivage des fonds réunis; le rôle des associations dans la préservation du patrimoine lié à la grande pêche interroge la démarche de patrimonialisation engagée au cours d'un tel projet. La durée totale des expositions - 6 mois à Rennes et Saint-Brieuc d'octobre 2013 à avril 2014, puis 4,5 mois à Saint-Malo et Granville de fin juin à début novembre 2014 - a nécessité un important travail de recherche et de régie, et donc des frais supplémentaires, afin de pouvoir satisfaire aux exigences des prêteurs et organiser la rotation des collections notamment en arts graphiques. 
20 Un travail original et inédit a été mis en place dans la muséographie autour des productions audiovisuelles. Deux créations méritent particulièrement d'être signalées :

21 - deux films d'animation, La pêche sédentaire et La pêche errante, réalisés par la société Vivement lundi!, société rennaise reconnue pour son expertise en matière de cinéma d'animation au niveau national : l'utilisation des gravures de Duhamel du Monceau et leur animation est un bon exemple de valorisation de documents authentiques, tout en permettant une très bonne réception du public de par l'approche très pédagogique, simple mais efficace ;

22 - une borne multimédia sur les havres de pêche, confrontant ressources d'archives, photographies anciennes et contemporaines de sites archéologiques fouillés à TerreNeuve. Elle est venue valoriser un travail scientifique inédit et a été mise en ligne sur Internet à l'issue de l'exposition.

\section{Collaborer pour enrichir la recherche et les collections... ainsi que les professionnels}

Développée par le chef de projet, une base de données a été mise en place avec l'expérimentation pour le musée du logiciel de gestion, Filemaker Pro. La base ainsi créée est un outil de gestion de l'ensemble des contenus présentés (matériel et immatériel), permettant le suivi de l'ensemble des questions liées à la régie des œuvres, à la gestion des droits, aux questions techniques relatives au soclage ou aux impératifs de conservation préventive... Elle recense aujourd'hui, sur des fiches individuelles, plus de 3400 items dotés d'une photographie documentaire.

De prise en main relativement simple et d'une grande adaptabilité, cet outil a permis une gestion facilitée des projets d'exposition rassemblant un grand nombre de fonds.

Cette expérience concluante a convaincu le musée de se doter de cet outil, étendu aux nouveaux projets de manière collaborative. Par ailleurs, cette coproduction a favorisé une progression individuelle et collective des équipes des musées concernés, par la mutualisation du temps et des compétences, la formation interne et la mise en réseau des institutions, simplifiant l'émergence de projets partenariaux. La seule limite - mais de taille - concerne la valorisation de cette base, qui reste un document de travail et n'a pu être mise en ligne. Les problématiques de droits, tant sur les collections privées que publiques et surtout les documents audiovisuels, le travail de suivi et les coûts afférant dépassaient de loin les capacités du projet.

Le travail collaboratif autour de ce projet d'exposition a aussi permis la réalisation d'un ouvrage de 160 pages, richement illustré et documenté, rassemblant les regards des deux expositions. Il constitue aujourd'hui un livre de référence sur la pêche à Terre-Neuve et sur son patrimoine, édité à 3000 exemplaires et qui a connu une bonne diffusion dans les musées, par le réseau de diffusion de l'éditeur mais aussi à Terre-Neuve et Saint-Pierreet-Miquelon ${ }^{4}$. 


\section{Co-produire pour croiser les publics et permettre le rayonnement des musées}

L'exposition Terre-Neuve/Terre-Neuvas est une exposition qui a rencontré son public, avec une fréquentation de très bon niveau par rapport aux fréquentations habituelles des sites respectifs, près de 70000 visiteurs sur les quatre sites, dont 32000 à Rennes, 19000 à Saint-Malo, 12000 Saint-Brieuc et 6500 à Granville.

Les enquêtes menées auprès des publics, tant par la mise en œuvre d'un questionnaire administré (plus de 500 questionnaires administrés) que par l'analyse des commentaires des livres d'or (dont un livre numérique avec plus de 900 commentaires) attestent aussi d'une importante satisfaction des visiteurs (les termes "beau" , "émouvant"; "fierté" , "notre patrimoine" reviennent régulièrement); de même, les objectifs poursuivis en termes de cible public ont été atteints, avec des publics famille importants, et souvent la participation de trois générations au sein d'un même groupe. Les visites intergénérationnelles sont ainsi venues conforter notre démarche patrimoniale et s'intégrer dans nos propres actions de médiation sur un sujet en voie de patrimonialisation, entre mémoire et histoire.

Les dispositifs audiovisuels et numériques sur place, donnant accès à des contenus complémentaires et faciles d'accès, ont eux aussi été très utilisés, particulièrement par les jeunes, La fréquentation du site Internet dédié www.terreneuve.terreneuvas.fr n'est, elle, pas connue, en raison d'une défaillance technique du prestataire, ce qui est dommageable pour évaluer l'intérêt de mise en ligne des contenus scientifiques.

Dans l'atelier pédagogique les élèves retrouvent l'origine de la morue et du cabillaud pané en naviguant avec un équipage terre-neuvier.

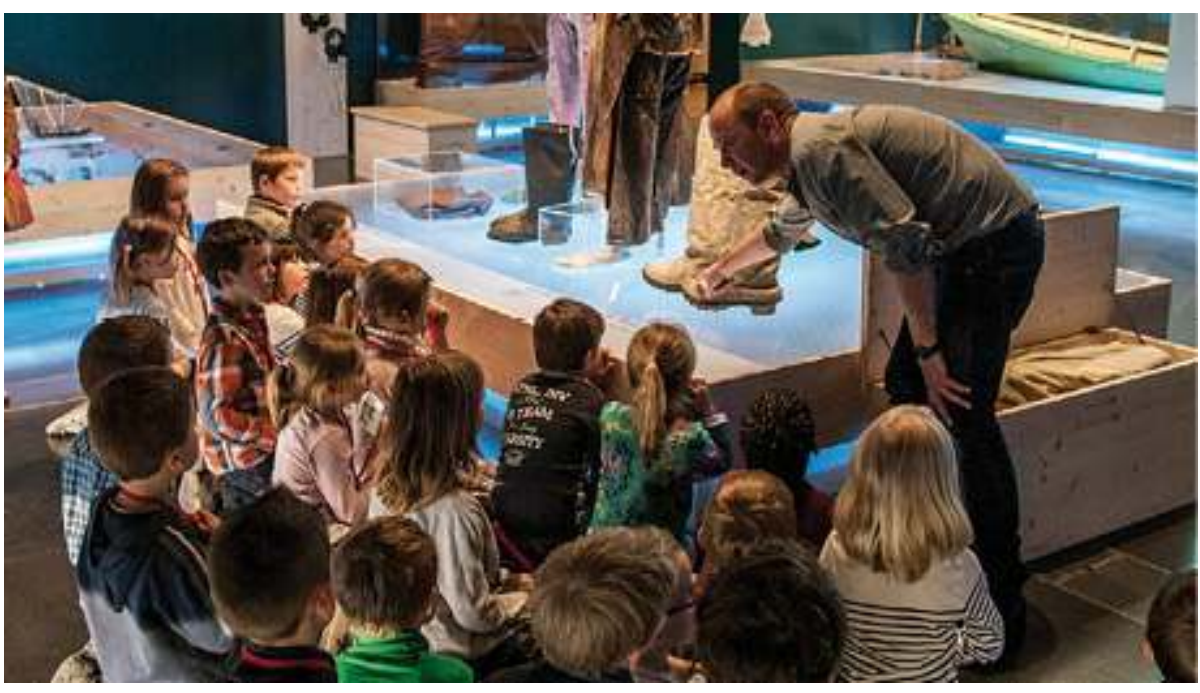

(c) musée de Bretagne/A. Amet

L'exposition Terre-Neuve/Terre-Neuvas aura aussi été l'occasion d'éprouver une nouvelle manière d'intégrer "la médiation" au montage d'une exposition. Ce souhait d'une meilleure prise en compte du confort (intellectuel et physique) du public des expositions, que les médiateurs expérimentent régulièrement au contact des visiteurs, s'est manifesté par une formalisation des interventions dans les différentes étapes du montage. 
31 Trois musées bénéficiaient d'un chargé de médiation sur l'exposition. Ce groupe projet "médiation" était animé par un chargé de coordination des actions de médiation basé au musée de Bretagne et intégré à l'équipe projet. Courroie de transmission avec les différents services des publics, ce positionnement d'un médiateur sur la quasi-totalité des phases pré-opérationnelles s'est avéré bénéfique. Plusieurs réunions des médiateurs référents des musées partenaires auront été organisées pour porter et suivre les souhaits et les remarques de la médiation en direction du commissariat général de l'exposition qui s'est montré réactif sur les questions relatives aux publics.

Plusieurs supports de médiation ${ }^{5}$ ont ainsi été créés pour une large accessibilité : malle pédagogique autour des vêtements, morue tactile, parcours adapté, jeu de l'oie et activités famille... La coproduction a ainsi permis la mutualisation d'outils de médiation que les petites structures n'auraient pas eu la possibilité de réaliser.

\section{Du temps, des moyens financiers et humains... et beaucoup d'énergie à déployer}

Ce projet a été très lourd à porter et réaliser, tant pour la maîtrise d'ouvrage que pour les trois autres musées impliqués, et ce en raison de plusieurs problématiques.

De manière globale, la co-production en musée de société, si elle permet la mutualisation de la recherche scientifique et la conception, ainsi que certains frais (scénographie, mobilier, fournitures...), ne supprime pas la charge de montage, de régie technique et des collections, très forte avec quatre lieux investis et une double itinérance.

À cela s'ajoutent plusieurs facteurs :

- le changement de direction au musée de Bretagne, intérim, reprise du commissariat ;

- la sous-estimation globale de la charge de travail en matière de coordination et de production, qui a nécessité de nombreux ajustements au cours du projet ;

- la volonté de conserver un fonctionnement collaboratif réel ;

- l'implication très forte des équipes scientifiques des musées partenaires, parfois dépendants pour la production d'équipes municipales non intégrées au musée et donc à mobiliser.

Outre la mobilisation des équipes permanentes - conservation, technique, communication, action culturelle et médiation, administration et juridique... - les musées, et particulièrement le musée de Bretagne ont dû faire appel au recrutement de contractuels, et mobiliser des ressources complémentaires, notamment de stages longs, partenariat avec l'université (évaluation). Mener un tel projet d'ambition régionale et itinérante sur plusieurs années, c'est donc disposer de moyens supplémentaires des équipes permanentes, estimés à environ 80 mois de travail (dont un chef de projet sur 24 mois).

41 L'affirmation d'un leadership d'organisation pour le pilotage global du projet et la conduite d'opération s'est-elle trouvé confortée par la mise en place de la conventioncadre initiale et la position "tête de réseau" du musée de Bretagne, son expertise ancienne en région dans ce type de production.

2 Régulièrement en effet, depuis ces 30 dernières années, le musée de Bretagne a mené des actions volontaristes de production d'expositions itinérantes. Pendant longtemps, le bras 
armé de ces productions a été l'association Buhez, qui a notamment facilité la mise en œuvre opérationnelle des projets, avec un engagement fort des conservateurs de Bretagne, particulièrement du musée.

Avec le projet Terre-Neuve/Terre-Neuvas, Pascal Aumasson, alors directeur du musée de Bretagne s'est inscrit dans cette tradition de rayonnement régional du musée de Bretagne, qui rejoignait aussi les politiques de rayonnement et de coopération de Rennes Métropole, particulièrement avec Saint-Malo. En revanche, le suivi administratif et financier a été beaucoup plus complexe, en raison de l'organisation en régie, des procédures de commandes publiques et d'annualisation budgétaire, difficilement compatibles avec une gestion de projet pluriannuelle.

Concernant le bilan global du projet, il faut donc à nouveau souligner son caractère très positif, tant sur le plan de la coopération scientifique et muséale que du point de vue des publics. Les difficultés ont aussi été nombreuses (calendrier, financement, marchés infructueux...) et le travail collectif, doublé d'une motivation et mobilisation très forte des équipes a permis de trouver des solutions pour "accoucher" de ce projet d'ampleur.

Préparatifs de l'itinérance de l'exposition : constats d'état, mise en caisse des collections et du mobilier d'exposition

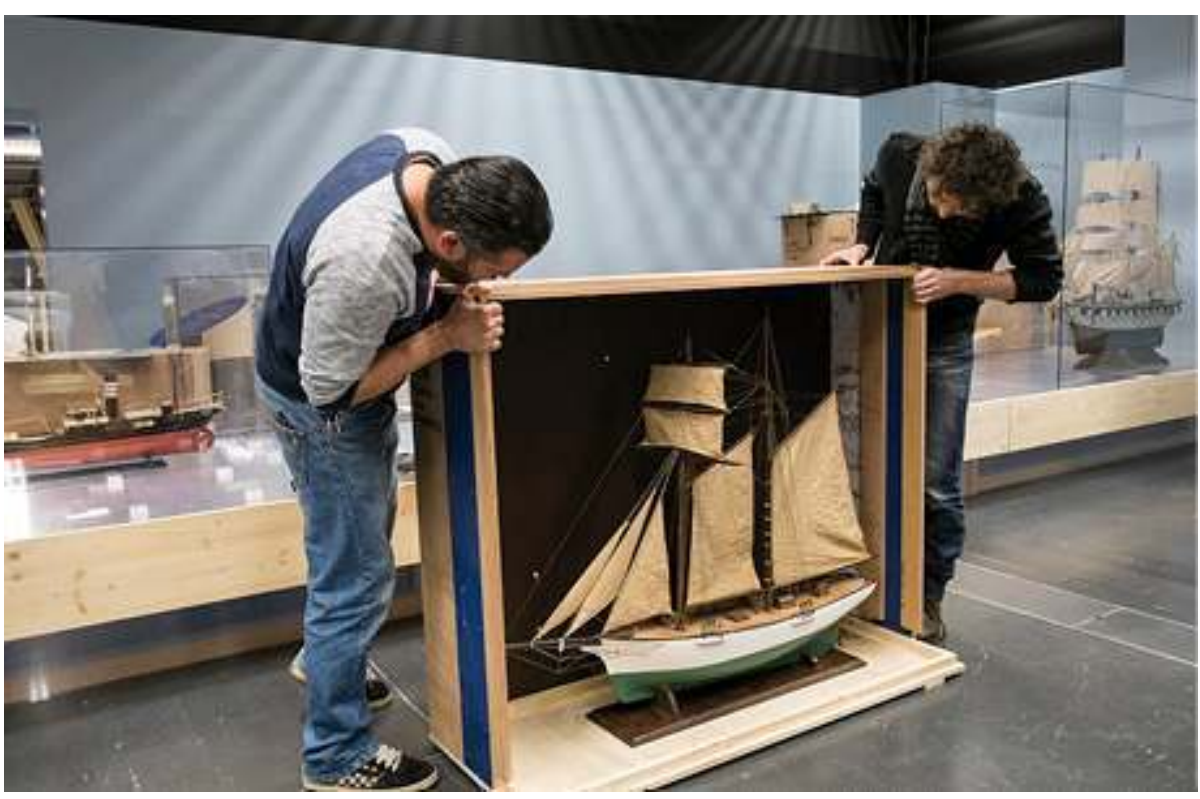

(C) Musée de Bretagne/A. Amet

Quelles sont aujourd'hui les perspectives ouvertes?

- une ingénierie en matière de production d'exposition, avec la montée en compétence de l'ensemble des équipes sur ce type de projet (planification, scénographie, médiation...). Le musée de Bretagne a prouvé sa capacité à mener de tels projets, qui nécessitent toutefois une meilleure consolidation, bien en amont, de la construction financière du projet, et donc un engagement politique précoce;

47 - une mise en réseau des musées en région, appelée à renouveler des projets : ainsi, en 2015 ont déjà été engagés avec Saint-Brieuc, deux collaborations, autour de Migrations , production du musée de Bretagne et Bretonnes, autre projet partenarial. Les élus de Rennes Métropole portent fortement la coopération avec Saint-Malo et de nouveaux projets sont à imaginer à un horizon 2018-2020 ; 
48 49 projet temporaire dans lequel se sont fortement investis les collectivités publiques, en premier lieu la métropole de Rennes, la région Bretagne et le ministère de la Culture, acteurs majeurs du soutien aux musées en Bretagne ;

51 . les musées labellisés doivent être les institutions de référence pour porter un discours scientifique, collections à l'appui, sur la Grande Pêche : suivi de collections à mener dans les années à venir, pour assurer l'enrichissement des fonds publics; suivi de la sauvegarde de la mémoire orale.

\section{BIBLIOGRAPHIE}

Benaiteau, C., Benaiteau, M., Berthon, O. et Lemonnier, A. Concevoir et réaliser une exposition - Les métiers, les méthodes. Paris : Eyrolles, 2012, 175 p.

Chanas, C., Chartrain, M., Liborio, M., Petout, P. et Renault, E. (dir.), Terre-Neuve/Terre-Neuvas : exposition. Rennes, Saint-Brieuc, Saint-Malo, Granville, musée de Bretagne, musée d'Art et d'Histoire, musée d'Histoire, musée d'Art et d'Histoire, 2013-2014. Rennes : musée de Bretagne, 2013. 167 p.

Steiner, C. et Courvoisier, F.-H. Les impacts des écrans tactiles sur les visiteurs dans les musées, La Lettre de l'OCIM, n 160, juillet-août 2015, pp. 10-16.

Draper, J.-L., Clubb, B. et Yarrow, A. Bibliothèques publiques, archives et musées : tendances en matière de collaboration et de coopération. La Haye : Fédération internationale d'associations de bibliothèques et d'institutions (IFLA), 2008, 60 p. (Collection Rapports professionnels de l'IFLA, n -109).

Les pratiques de mutualisation et de coopération dans le secteur culturel - Pourquoi ? Quoi ? Avec qui? Comment? Chambre Régionale de l'Économie Sociale et Solidaire Provence-Alpes-Côte d'Azur (CRESS PACA), Marseille : Conseil régional Provence-Alpes-Côte d'Azur, 2011, 15 p.

\section{NOTES}

1. À noter que le label d'intérêt national est assez rarement accordé à des expositions d'histoire et de société. Il est assorti d'une subvention notable du ministère de la Culture.

2. Le musée de Bretagne est un équipement métropolitain situé aux Champs Libres à Rennes.

3. Ces musées présentent dans leur parcours permanent une section consacrée à l'histoire de la Grande Pêche, dans une muséographie déjà datée et essentiellement centrée sur le bassin d'armement local. 
4. Terre-Neuve/Terre-Neuvas, catalogue collectif (double exposition itinérante 2013-2014) Musée de Bretagne, Rennes (19 octobre 2013-19 avril 2014), musée d'Histoire, Saint-Malo, musée d'Art et d'Histoire, Saint-Brieuc musée du vieux Granville, Granville, Trouville-sur-Mer, Illustria, 2013...

5. Ces supports de médiation ont été financés pour partie par le biais du mécénat et avec le bénévolat des membres de l'association des amis du musée (AMEBB).

\section{RÉSUMÉS}

À partir d'une expérience menée au musée de Bretagne, cette contribution met en lumière les enjeux de la coproduction d'expositions - notamment du point de vue du public et de l'accessibilité -, les avantages en termes de mutualisation des coûts et de l'ingénierie mais aussi les difficultés rencontrées lors des montages financiers et juridiques et au moment des choix scientifiques et culturels.

\section{INDEX}

Mots-clés : musée de Bretagne, coproduction

\section{AUTEUR}

\section{CÉLINE CHANAS}

conservateur en chef du patrimoine, directrice du musée de Bretagne à Rennes

c.chanas@leschampslibres.fr 